EPSC Abstracts

Vol. 14, EPSC2020-692, 2020

https://doi.org/10.5194/epsc2020-692

Europlanet Science Congress 2020

(C) Author(s) 2021. This work is distributed under

the Creative Commons Attribution 4.0 License.

\title{
Characterization of the atmosphere of TRAPPIST-1 e with JWST and ELT
}

\author{
Fabian Wunderlich ${ }^{1,2}$, Markus Scheucher ${ }^{1,2}$, Mareike Godolt ${ }^{2}$, John Lee Grenfell ${ }^{1}$, Franz Schreier ${ }^{3}$, \\ P. Christian Schneider ${ }^{4}$, David J. Wilson ${ }^{5}$, Alejandro Sánchez López ${ }^{6}$, Manuel López Puertas ${ }^{7}$, and \\ Heike Rauer ${ }^{1,2,8}$ \\ ${ }^{1}$ Institut für Planetenforschung, Deutsches Zentrum für Luft- und Raumfahrt, Rutherfordstraße 2, Berlin, Germany \\ (fabian.wunderlich@dlr.de) \\ ${ }^{2}$ Technische Universität Berlin, Zentrum für Astronomie und Astrophysik, Physik, Berlin, Germany \\ ${ }^{3}$ Institut für Methodik der Fernerkundung, Deutsches Zentrum für Luft- und Raumfahrt, Oberpfaffenhofen-Wessling, Germany \\ ${ }^{4}$ Hamburger Sternwarte, Gojenbergsweg 112, 21029 Hamburg, Germany \\ ${ }^{5}$ McDonald Observatory, University of Texas at Austin, Austin, TX 78712, USA \\ ${ }^{6}$ Sterrewacht Leiden, Universiteit Leiden, Postbus 9513, 2300 RA Leiden, The Netherlands \\ ${ }^{7}$ Instituto de Astrofísica de Andalucía (IAA-CSIC), Glorieta de la Astronomía s/n, 18008 Granada, Spain \\ ${ }^{8}$ Institut für Geologische Wissenschaften, Freie Universität Berlin, Malteserstr. 74-100, Berlin, Germany
}

The TRAPPIST-1 system is one of the most prominent targets for characterizing the atmospheres of terrestrial planets in the near future. We model potential atmospheres of planet e, which lies well in the habitable zone of the star and could hold liquid water. However, during the extended pre-main sequence phase of TRAPPIST-1, the planets may have experienced extreme water loss, leading to a desiccated mantle.

We simulate dry and wet atmospheres of TRAPPIST-1 e using a newly developed photochemical model for planetary atmospheres, coupled to a radiative-convective model then calculate theoretical spectra to determine how distinguishable these scenarios could be.

The resulting atmospheric composition is used to compute cloud-free transmission spectra. From this we calculate the detectability of molecular features using the Extremely Large Telescope (ELT) and the James Webb Space Telescope (JWST).

\section{Introduction}

The nearby terrestrial planet TRAPPIST-1 e, orbiting the cool host star TRAPPIST-1, offers the opportunity for studying its atmosphere with next generation telescopes.

The stellar luminosity evolution of such cools stars is quite different to that of e.g. the Sun.

In particular the active pre-main sequence phase of the star can be extended and the stellar Ultra Violet (UV) radiation is high for about a billion years [1].

This could lead to a runaway greenhouse state on an ocean-bearing terrestrial planet and a loss of substantial amounts of planetary water vapour before the star enters the main sequence phase [2]. This study aims to determine whether it is feasible to distinguish between wet and dry surface conditions of TRAPPIST-1 e from atmospheric observations using low resolution spectroscopy with the JWST and high resolution cross-correlation spectroscopy with the ELT. 


\section{Methods}

We use the 1D climate-photochemistry model 1D-TERRA $[3,4]$ in order to simulate the climate and photochemical response from wet and dry surface conditions. The TRAPPIST-1 input spectrum is taken from [5].

For wet conditions we assume a relative humidity of $80 \%$ and calculate dry as well as wet deposition for all species considered in the model. We consider in total three scenarios. The first two scenarios assume a liquid ocean at the surface. Scenario 1 is a dead case with only volcanic fluxes. Scenario 2 is an alive case with volcanic and Earth-like biogenic fluxes. Scenarios 3 is without a surface ocean using a relative humidity of $1 \%$, no wet deposition and weaker dry deposition for $\mathrm{CO}$ and $\mathrm{O}_{2}$ compared to the wet scenarios 1 and 2. For all scenarios we then simulate $\mathrm{N}_{2}$ atmospheres with different amounts of $\mathrm{CO}_{2}$ ranging from 0.001 bar to 1 bar.

The simulated atmospheric composition and temperature profiles are used to predict potential transmission spectra of TRAPPIST-1 e. These spectra are then used to estimate the number of transits required to detect molecular features with JWST NIRSpec and ELT HIRES.

\section{Results}

For dry $\mathrm{CO}_{2}$-rich atmospheres a significant amount of $\mathrm{O}_{2}$ and $\mathrm{O}_{3}$ is produced abiotically $[6,7]$, due to the

low FUV/NUV ratio of TRAPPIST-1 [8]. However, the abundances of abiotic $\mathrm{O}_{2}$ and $\mathrm{O}_{3}$ are one order of magnitude lower than in those

runs with biogenic emissions. A detection of $\mathrm{O}_{2}$ or $\mathrm{O}_{3}$ will be challenging with JWST NIRSpec or ELT HIRES (see Fig. 1). 
TRAPPIST-1 e
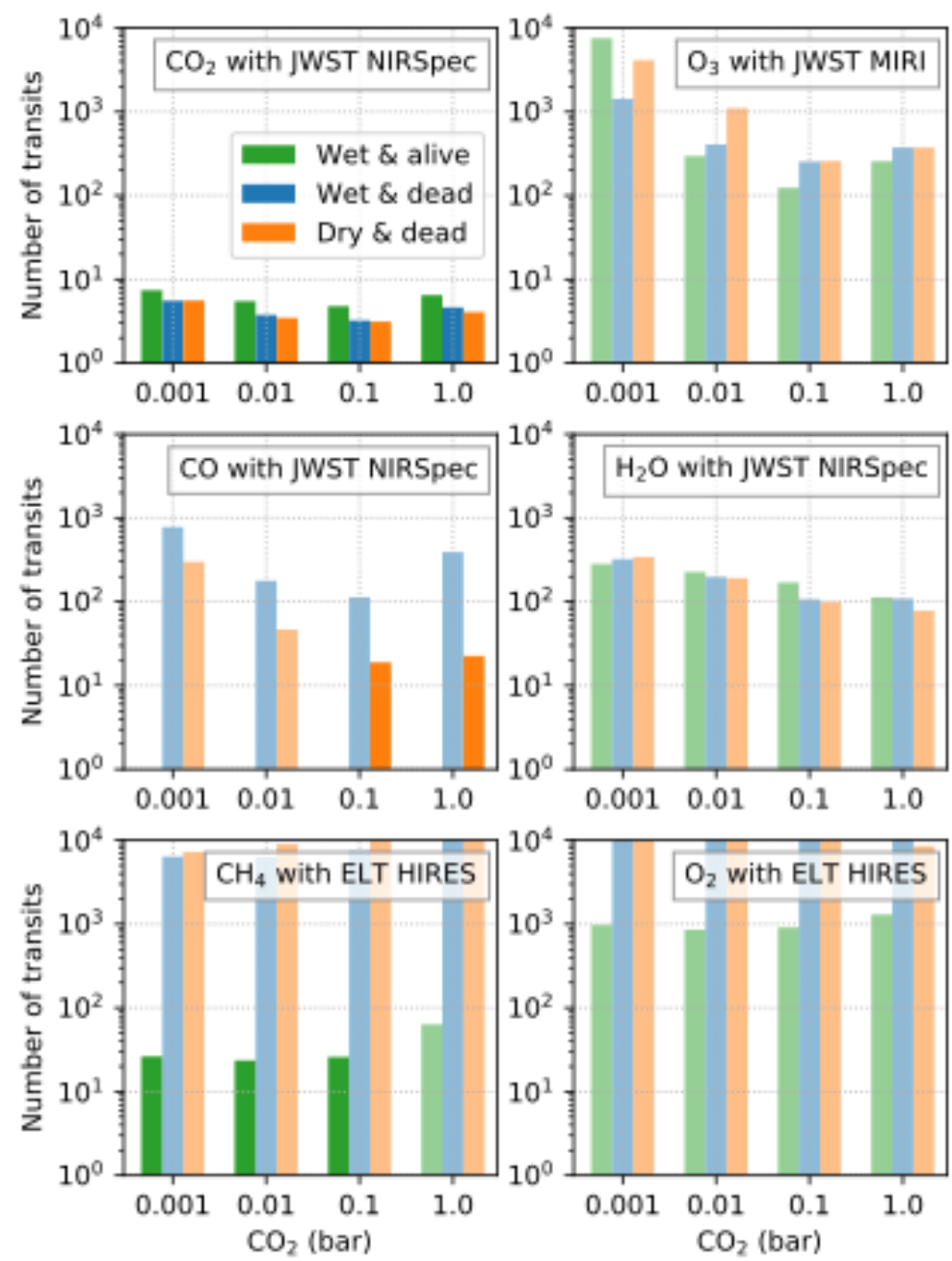

Figure 1: Number of transits required to reach a $\mathrm{S} / \mathrm{N}$ of 5 for $\mathrm{CO}_{2}$ at $4.3 \mu \mathrm{m}, \mathrm{O}_{3}$ at $9.6 \mu \mathrm{m}, \mathrm{CO}$ at $2.35 \mu \mathrm{m}$ and $\mathrm{H}_{2} \mathrm{O}$ at $1.4 \mu \mathrm{m}$ with JWST NIRSpec (upper and middle panel) and $\mathrm{CH}_{4}$ from 2.1 to 2.5 $\mu \mathrm{m}$ and $\mathrm{O}_{2}$ from 1.24 to $1.3 \mu \mathrm{m}$ with ELT HIRES (lower panel) in the atmosphere of TRAPPIST-1 e. Full filled bars: required number of transits is below or equal 30. Semi transparent bars: required number of transits is larger than 30. Figure from [4].

CO can be an indirect marker of an ocean, having concentrations enhanced by $\sim 100$ times on an ocean-less world with a $\mathrm{CO}_{2}$-rich atmosphere (see also $[9,10,11]$ ).

The detection of $\mathrm{CO}$ in the K-band might be feasible with JWST NIRSpec and ELT HIRES for dry surface conditions and $\mathrm{CO}_{2}$ partial pressure above 0.01 bar by co-adding several tens of transits.

Significant amounts of $\mathrm{CH}_{4}$ are only present in the simulated atmospheres with Earth-like biogenic flux. It has been shown that for planets around cool host stars, weaker destruction of $\mathrm{CH}_{4}$ leads to stronger spectral features of $\mathrm{CH}_{4}$ compared to the Earth around the Sun [12].

About 30 transit observation in the K-band are needed to detect $\mathrm{CH}_{4}$ with ELT HIRES for the wet \& alive case.

In conclusion, the three scenarios considered for TRAPPIST-1 e might be distinguishable by combining 30 transit observations with JWST NIRSpec and ELT HIRES in the K-band. The alive scenario, assuming Earth-like biogenic emissions, could be identified by the detection of $\mathrm{CH}_{4}$. The 
non-detection of $\mathrm{CO}$ would suggest the existence of a surface ocean. In turn, the detection of $\mathrm{CO}$ would suggest dry surface conditions (see more details in [4]).

\section{References}

[1] Baraffe, I., Homeier, D., Allard, F., \& Chabrier, G. 2015, A\&A, 577, A42.

[2] Luger, R., \& Barnes, R. 2015, AsBio, 15, 119.

[3] Scheucher, M., Wunderlich, F., Grenfell, J. L., et al. accepted, Ap].

[4] Wunderlich, F., Scheucher, M., Godolt, M., et al. accepted, ApJ.

[5] Wilson, D. J., Froning, C. S., Duvvuri, G. M., et al. submitted, ApJ.

[6] Selsis, F., Despois, D., \& Parisot, J.-P. 2002, A\&A, 388, 985.

[7] Harman, C. E., Schwieterman, E. W., Schottelkotte, J. C., \& Kasting, J. F. 2015, ApJ, 812, 137.

[8] Tian, F., France, K., Linsky, J. L., Mauas, P. J., \& Vieytes, M. C. 2014, EPSL, 385, 22.

[9] Gao, P., Hu, R., Robinson, T. D., Li, C., \& Yung, Y. L. 2015, Ap], 806, 249.

[10] Schwieterman, E. W., Reinhard, C. T., Olson, S. L., et al. 2019, Ap], 874, 9.

[11] Hu, R., Peterson, L., \& Wolf, E. T. 2020, Ap], 888, 122.

[12] Wunderlich, F., Godolt, M., Grenfell, J. L., et al. 2019, A\&A, 624, A49. 